\title{
MAXIMUM LIKELIHOOD ESTIMATION OF DIGITAL IMAGE CENTER
}

\author{
LU C'HUN-LIN \\ Purple Mountain Observatory, \\ Academia Sinica, \\ Nanjing 210008, China.
}

\begin{abstract}
A algorithm for digital image centering with the maximum likelihood method is described. The astrometric accuracy of this algorithm is similar to the two-dimensional Ciaussian fitting. For saturated images, this algorithm is better than the Gaussian fitting. With the maximum likelihood method, some systematic effect, such as coma and magnitude equation, can be reduced significantly.
\end{abstract}

\section{Introduction}

In modern astrometry, star positions are being determined by digital image analysis more and more commonly. besides automatic two-dimensional digital scanning of photographic plates, classical instruments, such as meridian circle, astrolabe, are also acquiring digital image of stars and determining their positions by digital algorithm. Several algorithms of digital image centering have been developed and extensively applied to photographic astrometry (van Altena \& Auer 1975, Chiu 1977, Auer \& van Altena 1978,Stetson 1979, Stone 1989, Platais 1991). Among them, the Gaussian fitting proved to be the most accurate one. However, the algorithm does have some disadvantages, e.g. it is not very good for centering faint images, the solution may diverges in certain cases, and it is the slowest algorithm (Stone 1989). To avoid of them, Platais(1991) offered the centering algorithm using the cubic spline technique. It has improved convergence for faint stars, but for the normal range of star image, its precision is $10-15 \%$ lower than the two-dimensional Gaussian fitting (van Altena 1991).

On the other hand, Gaussian fitting is a kind of least-square estimation, which is the optimal algorithm only under the condition of white noise. In practice, star image affected by a lot of factors, such as coma, magnitude equation, guiding error, blending etc. Based on these consideration, we introduced the maximum likelihood method into digital image centering. This paper will describe the method and its application.

\section{Algorithm}

In this paper, only the algorithm of two-dimension is discussed and it can be deduced to the case of one-dimension easily. Suppose the theoretical density distribution of an image is

$$
G=G\left(x, y ; x_{c}, y_{c}\right)
$$


where $x_{c}$ and $y_{c}$ are the center of the image. then the probability to obtain a measurement $D(x, y)$ of the image density is

$$
p(D \mid G)=\frac{1}{\sqrt{2 \pi} \sigma_{\mathrm{D}}} \exp \left\{-\frac{1}{2 \sigma_{\mathrm{D}}^{2}}\left[D(x, y)-G\left(x, y ; x_{c}, y_{c}\right)\right]^{2}\right\} .
$$

The likelihood function is

$$
L(\mathbf{D} \mid \mathbf{G})=\frac{1}{(2 \pi)^{n / 2} \sigma_{\mathrm{D}}^{n}} \exp \left[-\frac{1}{2 \sigma_{\mathrm{D}}^{2}}(\mathbf{D}-\mathbf{G}) \bullet(\mathbf{D}-\mathbf{G})\right],
$$

where $\mathbf{D}$ is the sample set of $n$ independent measurement values, being marginal distribution for the case of one dimension or density array for two dimension, $\mathbf{G}$ is the corresponding set of the theoretical values, and $\sigma_{\mathrm{D}}$ is the standard error of the measurement.

For a set of given measurement $\mathbf{D}, L(\mathbf{D} \mid \mathbf{G})$ can be considered as the function of $x_{c}, y_{c}$. According to the principle of maximum likelihood, the values $x_{c}$ and $y_{c}$ which make $L(\mathbf{D} \mid \mathbf{G})$ maximum are the maximum likelihood estimation of the image center coordinate. Therefore $x_{c}$ and $y_{c}$ can be obtained from the following equations:

$$
\begin{aligned}
& \frac{\partial \ln L(\mathbf{D} \mid \mathbf{G})}{\partial x_{c}}=0 \\
& \frac{\partial \ln L(\mathbf{D} \mid \mathbf{G})}{\partial y_{c}}=0
\end{aligned}
$$

From eqs.(4) and (5) we have

$$
\begin{aligned}
& \frac{\partial \mathbf{G}}{\partial x_{c}} \bullet \mathbf{D}-\frac{\partial \mathbf{G}}{\partial x_{c}} \bullet \mathbf{G}=0 \\
& \frac{\partial \mathbf{G}}{\partial y_{c}} \cdot \mathbf{D}-\frac{\partial \mathbf{G}}{\partial y_{c}} \cdot \mathbf{G}=0
\end{aligned}
$$

In practical application to density arrays of photographic star image obtained by PDS scanning, Gaussian function is usually adopted to represent the density distribution of star images. In this case, the second terms in eqs.(6) and ( 7$)$ equal to zero because the Gaussian function is even function, and we have

$$
\begin{aligned}
& \frac{\partial \mathbf{G}}{\partial x_{c}} \bullet \mathbf{D}=0 \\
& \frac{\partial \mathbf{G}}{\partial y_{c}} \bullet \mathbf{D}=0
\end{aligned}
$$

Solveing (8) and (9), we can obtain the image center $x_{c}$ and $y_{c}$.

It is difficult to solve eqs. (8) and (9) directly. We used a method similar to the calculation of convolution. To solve the equation, the model $G$ should be defined first, which need three parameters, that is, the half width $R$ and the approximate values of $x_{c}$ and $y_{c} . x_{c}$ and $y_{c}$ can be obtained by moment analysis. Then the second order moments $M_{x x}$ and $M_{y y}$ can be calculated. The smaller one between $M_{x x}$ and $M_{y y}$ is adopted as $R$.

Substituting $y_{c}$ and $R$ obtained with moment analysis into (8), searching for the $x_{c}$ which makes eq.(8) hold. Equation (9) can be solved similarly. 


\section{Astrometric accuracy}

Plates of Praesepe taken with the twin astrograph $(\mathrm{f}=300 \mathrm{~cm}, \mathrm{D}=40 \mathrm{~cm})$ at Purple Mountain Observatory(PMO) are used. The emulsion is Kodak 103a.O, with 15 minutes exposure. The image is saturated at about 9.5th magnitude. More than 300 stars were selected from Russell's catalog of Praesepe region (Russell 1976).

The plates were raster scanned using the PDS 1010MS of PMO, with an aperture of $20 \times 20 \mu \mathrm{m}$ and scanning step of $10 \mu \mathrm{m}$.

The digital images obtained by PDS scan are treated using both two-dimensional Gaussian fitting and the maximum likelihood method. To test the astrometric accuracy, plate reduction is performed using Russell's catalog. The stars are divided into three groups according to magnitude, each contains about 100 stars. The first group consists of bright stars with magnitude from 5.7 to 9.5 , in which most of the images are saturated. The second group includes stars with magnitude from 9.5 to 12.2. The third one is fainter stars with magnitude 12.2 to 16.0. The reduction is carried out for each group as well as for all stars together with different plate models. It can be seen from the rms errors of plate solution that, for well-formed images, both algorithms give similar accuracies. But for saturated or under-exposured images, the accuracy obtained with the maximum likelihood method is higher than the one by Gaussian fitting.

solutions are given in Table 1, where 'square' means the plate model with $\mathrm{M}$ is maximum likelihood method and $\mathrm{G}$ is two-dimensional Gaussian fitting. The number after $\mathrm{G}$ and $\mathrm{M}$ denotes the groups, no number means all stars. The unit is in $\mu \mathrm{m}$.

It is noticed that, for simpler plate models, in which only terms related to coordinates are included, the rms errors of maximum likelihood method is obviously smaller, but for the optimum model chosen with the Eichhorn-Williams criterion (Eichhorn \& Williams 1963), in which the terms related to magnitude, e.g. coma and equation, are included, the differences between the rms errors of the two methods are not very significant. It is because that some effects depended on magnitude have been reduced greatly in the maximum likelihood method, so some terms, which are included in the plate models for Gaussian fitting, are not significant and not included in the plate models.

TABLE 2. Coefficients of coma terms

\begin{tabular}{llrr}
\hline Plate & Method & \multicolumn{2}{c}{ Coefficients (in 10 } \\
\cline { 3 - 4 } & & \multicolumn{2}{c}{$x$} \\
\hline P1 & M & $-1.42 \pm 1.27$ & $2.76 \pm 1.64$ \\
& G & $-9.91 \pm 1.30$ & $-7.97 \pm 1.66$ \\
& & & \\
P2 & M & $-5.36 \pm 1.49$ & $-3.50 \pm 1.83$ \\
& G & $-15.70 \pm 1.50$ & $-18.50 \pm 1.88$ \\
& & & \\
P3 & M & $-7.71 \pm 1.24$ & $-5.73 \pm 1.57$ \\
& G & $-11.20 \pm 1.17$ & $-9.87 \pm 1.50$ \\
\hline
\end{tabular}

In Table 1, the coefficients of radial coma terms are listed. The half width $R$ is used as magnitude parameters, the unit of $R$ and $x, y$ is in $\mu \mathrm{m}$. It is shown that the effect of coma is almost eliminated by the maximum likelihood method. The magnitude equation is also weakened. 


\section{Conclusion}

The maximum likelihood method is used to conter digital image. The accuracy obtained with this method is similar to the two-dimensional Gaussian fitting for well-formed images but is higher for saturated images. One of the most attractive properties of the method is that it can significantly reduce some systematic effects related to magnitude. It is important because the spread of magnitudes of the reference stars will not in general cover the complete range of all stars to be positioned, although in the conventional practice significant magnitude dependent parameters can be found in plate solution, their extrapolation to fainter stars need not be valid.

As a kind of nonparametric approach, in principle, the maximum likelihood method will not diverge, no matter how the image is. Its speed of computation is much faster than two-dimensional Gaussian fitting.

The main disadvantage of the method is that it can not provide the information about the image structure and quality. A remedy is to obtain image structure parameters by moment analysis while defining image model (Stobie 1980). About the internal precision of computed coordinates, although it is theoretically possible to give an estimation, it is not feasible because of the complicated calculation.

\section{REFERENCES:}

Auer L.H., van Altena W.F.(1978),'Digital image centering. II',Astron. J. 83, $531-537$.

Chiu L.-T. G. (1977), 'Astrometric techniques with a PDS microdensitometer', Astron. J. 82, 842-848.

Eichhorn H., Williams C.A. (1963), 'On the systematic accuracy of photographic astrometric data', Astron. J. 68, 221-231.

Platais I. (1991), 'Astrometry with a PDS microdensitometer using cubic splines', Astron. Astrophy., 241, 656-662.

Russell J.L. (1976), The Astrometric Standard Region in Praesepe (M44), Ph.D. Dissertation, University of Pittsburgh

Stetson P.B. (1979), 'Photographic stellar Photometry with the PDS microdensitometer', Astron. J., 84, 1056-1066. Stobie R.S. (1980), 'Application of moments to the analysis of panoramic astronomical photographs',in Application of Digital Image Processing to Astronomy, SPIE, 264,p.208-212.

Stone R..C. (1989) 'A comparison of digital centering algorithms', Astron. J., $97,1227-1237$.

van Altena W.F. (1991), 'Photographic astrometry', Report on Astronomy, Vol.21A, 229.

van Altena W.F., Auer L.H. (1975), 'Digital image centering,I.', in C' de Jager, H. Nieuwenhuijzen(eds.), Image Processing Techniques in Astronomy, Reidel Dordrecht, p.411-418. 